\title{
Relationship between Food Addictive Behavior and Physical Activity in College-Aged Students
}

\author{
Stephen Bailey*, Carlie Huberman, Rebecca Bryant, Stephen Van Rij, Elizabeth Bailey \\ Elon University, United States \\ *Corresponding author: baileys@elon.edu
}

\begin{abstract}
Background: Food addiction is believed to play a role in the development of obesity. Acute exercise has been associated with reduced cravings for addictive substances. The purpose of this investigation is to determine if food addictive behavior is influenced by physical activity in college-aged students. Methods: 241 college-aged students (18-24 years of age; 55 male, 186 female) were recruited and completed the Yale Food Addiction Scale 2.0 (YFAS) and the International Physical Activity Questionnaire Short Form (IPAQ-S). Results: Only 7.9\% of participants were diagnosed with moderate (2.5\%) or severe (5.4\%) food addiction (FA). BMI was higher in participants with food addiction (No FA=24.1 $\pm 0.3 \mathrm{FA}=26.9 \pm 1.9, \mathrm{M} \pm \mathrm{SE}, \mathrm{p}=0.02$ ); however, no differences were found in vigorous (No FA=1851 \pm 251 MET-min/week, FA=1920 \pm 799 MET-min/week), moderate (No FA=777+107 MET-min/week, FA=869+409 MET-min/week), or walking (No FA=1262 \pm 139 MET-min/week, FA=2051+583 MET-min/week) activity. The distribution of participants with Low, Moderate, and High levels of physical activity was similar between No FA and FA. Participants with High (2.10 \pm 0.27 symptoms) levels of physical activity displayed more symptoms of food addictive behavior than participants with Moderate $(1.41 \pm 0.15$ symptoms) and Low (1.06 \pm 0.27 symptoms) levels $(\mathrm{p}=0.03)$. Conclusions: The results of this investigation suggest that high levels of physical activity may be associated with more symptoms of food addiction.
\end{abstract}

Keywords: addiction, physical activity, college-aged

Cite This Article: Stephen Bailey, Carlie Huberman, Rebecca Bryant, Stephen Van Rij, and Elizabeth Bailey, "Relationship between Food Addictive Behavior and Physical Activity in College-Aged Students." Journal of Physical Activity Research, vol. 2, no. 2 (2017): 68-72. doi: 10.12691/jpar-2-2-1.

\section{Introduction}

More than 1/3 of Americans (36.5\%) are obese. [1] While obesity has multifactorial origins, there has been some recent focus on the role of food addiction on the development on obesity, its maintenance, and lack of response to treatment. The premise behind food addiction is based on the idea that highly processed foods, with added fats and/or refined carbohydrates, may trigger an addictive-like response in some people. Results of investigations utilizing animal models support the food addition theory by showing that highly processed foods or their ingredients cause biological (change in dopaminergic activity in the brain) and behavioral (bingeing, failure to respond to negative consequences) responses typically seen is substance addiction. [2,3,4]

The Yale Food Addiction Scale, Version 2 (YFAS 2.0) is based on the criteria for substance-related and addictive disorders from the Diagnostic and Statistical Manual of Mental Disorders, version 5 (DSM-5) and is the only validated instrument designed to assess addictive-like eating behavior in humans. [5] Previous work with the YFAS has revealed that people with food addiction display similar behavioral characteristics to those seen in people with other types of substance addictions such as greater impulsivity and emotion dysregulation. [6,7] The similarity between food addiction and the addiction to other substances has also been strengthened by the work of Burger and Stice. [8] These authors found that people addicted to a specific highly processed food (ice cream) exhibited similar reward-related neural responses when anticipating and receiving the food as those seen under similar conditions in individuals addicted to other substances.

Acute exercise has been shown to reduce craving for various addictive substances (smoking, alcohol, opioids). $[9,10,11]$ Exercise training has also been show to improve outcomes related to treatment for addictive substances (alcohol, smoking stimulants) [12,13,14,15]. Furthermore, addiction to tobacco has been associated with lower levels of physical activity. [16] The specific physiologic mechanisms that facilitate the positive impact of physical activity on addictive behaviors are unclear; however, it appears that the positive psychological adaptations of reduced anxiety and depression associated with increased physical activity are important to the phenomenon. Given that elevated levels of depression and anxiety have been associated with negative eating behavior in young and middle-aged adults [17], these findings suggest that physical activity may play an important role in regulating food addictive behavior. The purpose of this investigation was to determine if there is a relationship between level of physical activity and addictive eating behavior in collegeaged adults. It was hypothesized that higher levels of 
physical activity will be associated with a lower incidence of food addiction and fewer symptoms of food addictive behavior.

\section{Methods}

Subjects: College-aged students $(n=241)(18-24$ years of age; 55 male, 186 female) were recruited via email, social media, and word of mouth from university and community college settings and asked to complete the Yale Food Addiction Scale (YFAS) 2.0 and the short version of the International Physical Activity Questionnaire (Last 7-Days) (IPAQ-S) using an on-line platform. While completing the on-line questionnaires subjects also provided demographic information including age, sex, height, and weight. Body mass index (BMI) was calculated from self-reported height and weight.

Yale Food Addiction Scale 2.0: The YFAS 2.0 reflects the assessment of addictive-like eating behavior based on the substance related and addictive (SRAD) diagnostic criteria in the DSM-5. [5] The YFAS specifically documents the consumption of foods (high fat and refined carbohydrate) most associated with food cravings and binge eating. The YFAS 2.0 is scored in two ways: a) a continuous symptom count that reflects the number of diagnostic criteria met by the participant and b) by identifying the presence of food addiction based on the number of symptoms and clinically significant impairment or distress. The YFAS 2.0 identifies up to 11 SRAD diagnostic indicators of food addiction. Each of the 11 diagnostic criteria was considered to be met if one or more of the relevant questions for each criterion met the predetermined threshold. When identifying food addiction, the YFAS 2.0 places the individual into a mild (2 to 3 symptoms), moderate (4-5 symptoms), or severe (6 or more symptoms) category of food addiction.

IPAQ-S: The IPAQ-S is a self-report measure of physical activity (PA) and sitting time (sedentary behavior) [18]. It is a seven-day recall questionnaire that chronicles information on the time (duration) and frequency of walking, the time spent in moderate intensity, and vigorous intensity activity, as well as the time spent being sedentary. The total amount of PA for each participant is calculated as the metabolic equivalent (MET) minutes per week (MET-min/week). Subjects are categorized into low, moderate, and high levels of PA based on the number of MET-min/week.

Data Analysis: Subjects were categorized by food addiction, food addictive symptoms, and level of physical activity. First subjects were identified as being or not being food addicted according to the procedures described by Gearhardt and colleagues. [5] Differences between the groups in BMI and amount of physical activity were assessed using t-tests with a Bonferroni correction. Subjects were also categorized by the number of food addictive symptoms they displayed and placing them in to the previously described categories (none, mild, moderate, and severe). Differences between groups in level of physical activity were assessed using Chi-square (4 X 3) and one-way ANOVA.

Subjects were also categorized into low, moderate, and high levels of PA. The distribution of subjects within each category with and without food addiction was compared using Chi-Square analysis and the number of food addiction symptoms within each category was assessed using a 1X3 ANOVA and appropriate post-hoc analysis between specific cell means. Significance level for all analyses was set $a$ priori at $\mathrm{p}<0.05$.

\section{Results}

Impact of Food Addiction on Physical Activity: Nineteen of 241 (7.9\%) participants were identified as having moderate $(2.5 \%)$ or severe $(5.4 \%)$ food addiction. BMI was higher in participants with food addiction (FA) compared to those without FA (No FA) (No FA=24.1 \pm 0.3 , $\mathrm{FA}=26.9 \pm 1.9 ; \mathrm{p}=0,02)$; however, the presence-or absence of FA did not influence the level of total, vigorous, moderate, or walking activity reported by participants (Table 1; Figure 1).

Table 1. Total Amount of physical activity reported (MET Minutes/wee) when categorized by presence of food addiction and by the number of food addictive symptoms

\begin{tabular}{|c|c|}
\hline & $\begin{array}{l}\text { Level of Physical Activity } \\
\text { (MET-Minutes/week) }\end{array}$ \\
\hline \multicolumn{2}{|l|}{ Categorized by Food Addiction } \\
\hline Non-Addicted $(n=222)$ & $3890 \pm 289$ \\
\hline Addicted $(\mathrm{n}=19)$ & $4841 \pm 1098$ \\
\hline \multicolumn{2}{|c|}{ Categorized by Number of Food Addiction Symptoms Displayed } \\
\hline None $(0-1)(n=162)$ & $3780 \pm 358$ \\
\hline Mild (2-3) $(n=36)$ & $4314 \pm 644$ \\
\hline Moderate (4-5) $(n=18)$ & $3642 \pm 668$ \\
\hline Severe $(6-11)(n=25)$ & $4845 \pm 856$ \\
\hline
\end{tabular}

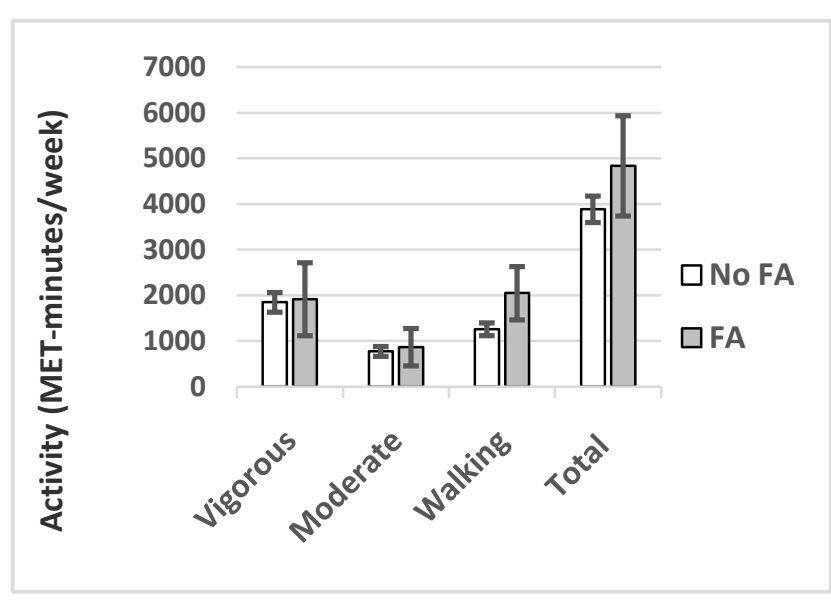

Figure 1. Differences in physical activity between participants without food addiction (No FA) and with food addiction (FA)

Influence of Food Addictive Symptoms on Physical Activity: While only $7.9 \%$ of participants displayed all of the criteria for food addiction, 32.9\% of participants displayed 2 or more food addictive symptoms. When using the criteria provided by Gearhardt and colleagues, $14.9 \%$ had a mild (2-3), $7.5 \%$ had a moderate (4-5), and $10.4 \%$ had a severe (6-11) number of food addictive symptoms. The frequency of presence of each symptom is provided in Table 2. Participants most frequently indicated that they had a desire to quit or have made numerous attempts to quit eating a particular food (27\%), have 
experienced withdrawal symptoms when discontinuing eating a particular food (23.7\%), have spent excessive amounts of time trying to get access to a food (22.4\%), or have consumed an amount of the food in excess of what was intended (21.2\%). No differences in self-reported physical activity level were seen when subjects were categorize by the number of food addictive symptoms displayed (Table 1).

Table 2. Diagnostic Indicators/Symptoms of Food Addiction and Prevalence in Sample Population $(n=241)$

\begin{tabular}{|l|r|}
\hline & Met Criteria \\
\hline $\begin{array}{l}\text { Substance taken in larger amount and for longer period } \\
\text { than intended }\end{array}$ & $51(21.2 \%)$ \\
\hline $\begin{array}{l}\text { Persistent desire or repeated unsuccessful attempts at } \\
\text { quitting }\end{array}$ & $65(27.0 \%)$ \\
\hline Much time/activity to obtain, use, recover & $54(22.4 \%)$ \\
\hline $\begin{array}{l}\text { Important social, occupational, or recreational activities } \\
\text { given up or reduced }\end{array}$ & $27(11.2 \%)$ \\
\hline $\begin{array}{l}\text { Use continues despite knowledge of adverse } \\
\text { consequences }\end{array}$ & $34(14.1 \%)$ \\
\hline $\begin{array}{l}\text { Tolerance (marked increase in amount; marked decrease } \\
\text { in effect }\end{array}$ & $27(11.2 \%)$ \\
\hline $\begin{array}{l}\text { Characteristic withdrawal symptoms; substance taken to } \\
\text { relieve withdrawal }\end{array}$ & $57(23.7 \%)$ \\
\hline Continued use despite social or interpersonal problems & $22(9.3 \%)$ \\
\hline $\begin{array}{l}\text { Failure to fulfill major role obligation (e.g., work, school, } \\
\text { home) }\end{array}$ & $18(7.5 \%)$ \\
\hline Use in physically hazardous situations & $27(11.2 \%)$ \\
\hline Craving, or a strong desire or urge to use
\end{tabular}

Impact of Level of Physical Activity on Food Addiction and Food Addictive Symptoms. When participants were categorized by level of self-reported physical activity, no differences were seen in the distribution of food addicted and non-food addicted participants $\left(X^{2}=0.93, \mathrm{p}=0.92\right)$. Similar findings were observed when participants were categorized by the number of food addictive symptoms displayed $\left(X^{2}=2.93\right.$, $\mathrm{p}=0.82$ ). Furthermore, no differences were found in BMI across physical activity categories (Low $=23.3 \pm 0.7$, Moderate $=25.2 \pm 0.6$, High=23.9 \pm 0.4$)$. In contrast, participants reporting high levels $(2.08 \pm 0.24)$ of physical activity displayed more food addictive symptoms than participants reporting low $(1.06 \pm 0.44)$ and moderate $(1.41 \pm 0.23)$ levels of physical activity ( $\mathrm{p}=0.49$, Figure 2$)$.

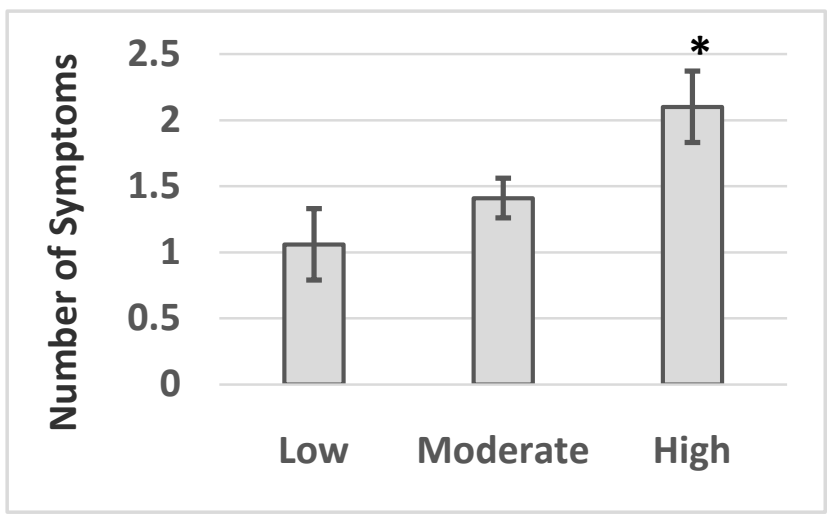

*Indicates difference $(\mathrm{p}<0.05)$ from Low and Moderate.

Figure 2. Number of food addiction symptoms across levels of physical activity

\section{Discussion}

In this investigation $7.9 \%$ of participants were found to have moderate $(2.5 \%)$ or severe $(5.4 \%)$ food addiction. Previous investigations that examined food addiction in a cross sectional sample of U.S. college-aged students have reported prevalence rates ranging from $10.3 \%$ [19] to $24 \%$ [7]. Prior to this investigation, authors examining the prevalence of food addiction in this population used the original YFAS and not the YFAS 2.0. It is possible that the differences in prevalence seen here are related to the different version of the YFAS applied. A potentially interesting difference between the prevalence of FA found by $\mathrm{Yu}$ and colleagues [19] as compared to that reported by Murphy and associates [7] is the platform used for data collection. Murphy and associates [7] collected data using a pencil and paper technique while $\mathrm{Yu}$ and colleagues [19] utilized an on-line platform. Since the results presented here are more consistent with those described by Yu et al [19], it seems that the data collection platform used is important to consider when interpreting results.

Gearhardt and colleagues developed the YFAS 2.0 in an effort to reflect the attributes of the DSM-5 regarding substance-related and addictive disorders (SRAD) [5]. When validating the new version of the YFAS (YFAS 2.0), they found that the rate of prevalence of FA in a broad sample of adults was greater when using the YFAS $2.0(15.8 \%)$ compared to the original YFAS (10.0\%) [5]. The fundamental difference between the YFAS and the YFAS 2.0 is that the YFAS only considers issues of dependence on a substance, while the YFAS 2.0 also assesses abuse of a substance. If our findings were consistent with Gearhardt and colleagues [5] then the percentage of college students with food addiction would be expected to be higher than those reported here and possibly in the higher end of the range discussed above for college-aged participants. While there are not any obvious demographic explanations for these differences, some insight may be gained when considering the distribution of students in this study reporting food addictive symptoms. In this investigation, $32.9 \%$ of participants displayed two or more symptoms of food addiction. Furthermore, only half of the participants displaying 6 or more symptoms of food addictive behavior were actually categorized as having food addiction. When using the YFAS 2.0, participants need to display at least 2 symptoms of food addictive behavior and report that food and eating have either caused significant problems in their life and/or caused them not to do important things in their life at least 2-3 times per week. It is possible that the way this age group interprets the questions related to abuse is unique and it seems obvious that this phenomenon should be further explored in future investigations.

In this investigation, participants most frequently indicated that they had: challenges related to quitting consumption of a particular food (27\%), withdrawal symptoms when deprived of the food (23.7\%), spent excessive time seeking access to the food (22.4\%), and consumed the food in excessive amounts (21.2\%) (Table 2). The frequency of symptom presence seen here when compared to that reported by Gearhardt and colleagues in a broader sample is not dramatically different; however, these authors 
reported much greater frequencies in the areas of failure of role obligation, social consequences, and use in physically hazardous situations [5]. Symptom frequencies reported in the current investigation appear to be more similar to those describe by Meule and associates in German college-aged students [20]; though we did observe higher frequencies of symptoms of withdrawal $(23.7 \%$ versus $14.7 \%)$ and time spent seeking the food of choice $(22.4 \%$ versus $11.0 \%$ ) than these authors. The impact of these differences in frequency of presence of symptoms of food addiction on the current question is not clear, but they do demonstrate the variability with which any given sample population could display food addictive behavior.

The primary purpose of this investigation was to examine the relationship between food addiction, food addictive behaviors, and physical activity. Since physical activity is associated with positive outcomes in other addictive substances, we hypothesized that more active participants would have a lower prevalence of food addiction and display fewer symptoms of food addictive behavior. It is clear that our data do not support this hypothesis. Contrary to our hypothesis, our data suggests that if anything there is a trend for participants with food addiction or more symptoms of food addictive behavior to report higher levels of physical activity (Table 1 and Table 2). While no statistical differences were seen in physical activity between non-addicted and food addicted participants, it appears that food addicted participants tended to report more walking activity than non-addicted participants (Figure 1). Upon close observation of the data it becomes obvious that there is large variability in the self-reported physical activity data, perhaps inhibiting the ability to see differences between groups. The physical activity data reported for the non-addicted group in this investigation is similar to magnitude and variability to that previously reported by Fernandes and colleagues [21]. While the IPAQ-S is consistently reliable, its validity is frequently called into question. Lee and colleagues concluded in a systematic review that the IPAQ-S overestimates physical activity by $84 \%$ [22]. As a result, it would be appropriate to repeat this investigation using an objective criterion of physical activity (e.g. accelerometers).

The one area where significant differences between the groups was observed was the number of food addictive symptoms displayed when participants were categorized by physical activity level (low, moderate, high). In this investigation, participants reporting a high level of physical activity displayed more symptoms of food addictive behavior than those in low and moderate physical activity groups (Figure 2). While this finding is consistent with the trend observed in self-reported physical activity, it is similarly contradictory to our original hypothesis. The explanation for this outcome is unclear; however, it is possible that participants with high levels of physical activity have a unique relationship with food that manifests as symptoms of food addiction.

The possible relationship between higher levels of physical activity and food addictive behavior may suggest to some that excessive amounts of exercise promote negative food behaviors. Exercise anorexia nervosa or anorexia athletica has been well documented in the literature and is associated with disordered eating behavior and excessive amounts of vigorous exercise. [23] It is unlikely that this disorder is manifesting itself in the data presented here for two reasons. These disorders, where excessive exercise is coupled with disordered eating, typically result in a lower than normal body weight. [23] No differences were observed in BMI across physical activity categories in this investigation and the average BMI for the high and low physical activity groups was in the normal range. [24] Furthermore, review of the physical activity data indicates that the tendency for the food addictive group to have higher levels of physical activity was driven by greater minutes of walking activity and not due to moderate or vigorous physical activity.

In summary, the presence of food addiction was not influenced by level of physical activity; however, people with the highest levels of self-reported physical activity displayed more symptoms of food addiction. These findings may have been limited by the variability present in the measurement tool used to assess physical activity and this phenomenon should be revisited using a more objective measure of physical activity. Applying these findings to the treatment of food addiction is tenuous. The strategy of increasing physical activity to positively impact addictive behavior and symptoms is probably most valuable in individuals with initially low levels of physical activity.

\section{References}

[1] Ogden CL, Carrol MD, Fryar CD, Flegal CM. Prevalence of Obesity Among Adults and Youth: United States, 2011-2014. NCHS Data Brief.

https://www.cdc.gov/nchs/data/databriefs/db219.htm. Accessed June 23, 2017.

[2] Avena NM, Bocarsly ME, Rada P, Kim A, Hoebel BG. After daily bingeing on a sucrose solution, food deprivation induces anxiety and accumbens dopamine/acetylcholine imbalance. Physiol Behav. 2008; 94(3): 309-315.

[3] Avena NM, Murray S, Gold MS. Comparing the effects of food restriction and overeating on brain reward systems. Exp Gerontol. 2013; 48(10): 1062-1067.

[4] Oswald KD, Murdaugh DL, King VL, Boggiano MM. Motivation for palatable food despite consequences in an animal model of binge eating. Int J Eat Disord. 2011; 44(3): 203-211.

[5] Gearhardt AN, Corbin WR, Brownell KD. Development of the Yale Food Addiction Scale Version 2.0. Psychol Addict Behav J Soc Psychol Addict Behav. 2016; 30(1): 113-121.

[6] Pivarunas B, Conner BT. Impulsivity and emotion dysregulation as predictors of food addiction. Eat Behav. 2015; 19: 9-14.

[7] Murphy CM, Stojek MK, MacKillop J. Interrelationships among impulsive personality traits, food addiction, and Body Mass Index. Appetite. 2014; 73: 45-50.

[8] Burger KS, Stice E. Frequent ice cream consumption is associated with reduced striatal response to receipt of an ice cream-based milkshake. Am J Clin Nutr. 2012; 95(4): 810-817.

[9] Bailey, S.P., Hall, E. E., Fareed, A. Effects of acute exercise on opiate and cigarette craving in methadone patients. Open Sports Sci J. 2011; 4(1): 22-26.

[10] Abrantes AM, Farris SG, Minami H, Strong DR, Riebe D, Brown RA. Acute Effects of Aerobic Exercise on Affect and Smoking Craving in the Weeks Before and After a Cessation Attempt. Nicotine Tob Res Off J Soc Res Nicotine Tob. May 2017.

[11] Roberts V, Gant N, Sollers JJ, Bullen C, Jiang Y, Maddison R. Effects of exercise on the desire to smoke and physiological responses to temporary smoking abstinence: a crossover trial. Psychopharmacology (Berl). 2015; 232(6): 1071-1081.

[12] Bernard P, Ninot G, Cyprien F, et al. Exercise and counseling for smoking cessation in smokers with depressive symptoms: a randomized controlled pilot trial. J Dual Diagn. 2015; 11 (3-4): 205-216. 
[13] Giesen ES, Deimel H, Bloch W. Clinical exercise interventions in alcohol use disorders: a systematic review. J Subst Abuse Treat. 2015; 52: 1-9.

[14] Manthou E, Georgakouli K, Fatouros IG, Gianoulakis C, Theodorakis Y, Jamurtas AZ. Role of exercise in the treatment of alcohol use disorders. Biomed Rep. 2016; 4(5): 535-545.

[15] Trivedi MH, Greer TL, Rethorst CD, et al. Randomized controlled trial comparing exercise to health education for stimulant use disorder: results from the CTN-0037 STimulant Reduction Intervention Using Dosed Exercise (STRIDE) Study. J Clin Psychiatry. 142017.

[16] Furlanetto KC, Mantoani LC, Bisca G, et al. Reduction of physical activity in daily life and its determinants in smokers without airflow obstruction. Respirol Carlton Vic. 2014; 19(3): 369-375.

[17] Quehl R, Haines J, Lewis SP, Buchholz AC. Food and mood: diet quality is inversely associated with depressive symptoms in female university students. Can J Diet Pract Res 23 2017: 1-5.

[18] Craig CL, Marshall AL, Sjöström M, et al. International physical activity questionnaire: 12-country reliability and validity. Med Sci Sports Exerc. 2003; 35(8): 1381-1395.
[19] Yu Z, Tan M. Disordered eating behaviors and food addiction among nutrition major college students. Nutrients. 2016; 8(11)

[20] Meule A, Müller A, Gearhardt AN, Blechert J. German version of the Yale Food Addiction Scale 2.0: Prevalence and correlates of "food addiction" in students and obese individuals. Appetite. 2017; 115: 54-61.

[21] Fernandes J, Arts J, Dimond E, Hirshberg S, Lofgren IE. Dietary factors are associated with coronary heart disease risk factors in college students. Nutr Res N Y N. 2013; 33(8): 647-652.

[22] Lee PH, Macfarlane DJ, Lam T, Stewart SM. Validity of the international physical activity questionnaire short form (IPAQ-SF) A systematic review. Int J Behav Nutr Phys Act. 2011; 8:115.

[23] Joy E, Kussman A, Nattiv A. 2016 update on eating disorders in athletes: A comprehensive narrative review with a focus on clinical assessment and management. Br J Sports Med. 2016; 50(3): 154-162.

[24] ACSM's Guidelines for Exercise Testing and Prescription. 10th ed. Philadelphia, PA: Wolters Kluwer; 2018. 